\title{
Fomento de la innovación dentro de las empresas a través de la gestión del conocimiento
}

\section{Promotion of innovation within companies through knowledge management}

María Auxiliadora Guerrero Bejarano, MBA

Universidad Internacional del Ecuador, Ecuador

Daniel Ricardo Silva Siu, MSc

Universidad ESAN, Perú

Autores para correspondencia: dsilva@pobox.com, maguerrerobe@uide.edu.ec

Fecha de recepción: 28 de Febrero de 2017 - Fecha de aceptación: 28 de Abril de 2017

\section{Resumen}

Las empresas, en estos días deben enfrentarse con mercados cada vez más influenciados por la evolución de la tecnología, lo que genera cambios en algunas industrias de manera casi permanente, razón por la que es imprescindible fomentar verdadera innovación que permita obtener ventajas competitivas que sean sostenibles en el tiempo; este análisis teórico permitirá obtener una visión clara de cómo la gestión del conocimiento en las empresas son fuente indiscutible de innovación.

Palabras clave: innovación; evolución de la tecnología; gestión del conocimiento

\begin{abstract}
Companies in these days must confront markets that are increasingly influenced by the evolution of technology, which generates changes in some industries almost permanently, which is why it is imperative to promote true innovation to obtain competitive advantages that are sustainable in time; This theoretical analysis will allow us to obtain a clear vision of how knowledge management in companies are an indisputable source of innovation.
\end{abstract}

Key words: innovation; evolution of technology; knowledge management 


\section{Gestión del Conocimiento}

La gestión del conocimiento es considerada como uno de los antecedentes más importantes de la innovación, estudiada desde hace ya algunas décadas, la gestión del conocimiento se define como la forma en la que las empresas manejan su información, el cómo se hace o Know How que permite que los nuevos miembros conozcan sus procesos y puedan realizar sus tareas adecuadamente (Darroch, 2005), existen estudios que afirman que habría mayor impacto sobre la innovación en la empresa, en la forma en la que se adquiere el conocimiento, así como la capacidad de actuación frente a él que en la manera en la que se promueve (Adams, Bessant, \& Phelps, 2006; Darroch \& McNaughton, 2002). La orientación al mercado también juega un papel importante en la gestión del conocimiento, el aprendizaje sobre el consumidor y sus costumbres presentarían oportunidades claras de innovación que el conocimiento de la organización se utilice de manera eficaz y eficiente para beneficio de la empresa (Jantunen, 2005).

Diferentes tipos de empresas manejan su gestión del conocimiento buscando generar diferentes tipos de innovación que les permitan mejorar resultados, en algunos casos procesos como el análisis de las mejores prácticas suelen ser mecanismos que permiten el desarrollo y adquisición de conocimiento que enriquecen los procesos y generan resultados esperados (Du Plessis, 2007; Gloet \& Terziovski, 2004; Jantunen, 2005; Jover, Montalvo, \& Ones, 2006).

Cuando el conocimiento se utiliza, el aprendizaje dentro de la organización se lleva a cabo, lo que representa mejoras en la cantidad de conocimiento disponible para la empresa; cuando una firma gestiona efectivamente el conocimiento se la considera una learning organization; se considera la gestión del conocimiento un guía filosófica dentro de las organizaciones que orienta la toma de decisiones estratégicas de los gerentes, razón por la que este constructo se estudia dentro del comportamiento organizacional. (Darroch, 2005; Darroch \& McNaughton, 2002). La gestión del recurso humano también podría influir en la gestión del conocimiento, así como en la innovación, razón por la que el estilo de liderazgo de los gerentes ha sido altamente relacionado con estas variables (Bassett-Jones, 2005; Kuo, Lai, \& Lee, 2011), es así que el alentar un comportamiento de trabajo en grupo que soporte la innovación y permirtir tiempo para dedicar al aprendizaje de nuevas formas de hacer las cosas habrían obtenido resultados mixtos en los casos que se han estudiado (Anantatmula, 2008; Blázquez Manzano, 2013); otros resultados demuestran que la codificación o generación de conocimiento explícito en base a datos de la organización no fomentaría la innovación, pero la capacidad de respuesta frente al conocimiento, al aprendizaje afecta positivamente la innovación (Darroch \& McNaughton, 2002)

Existen estudios que prueban que la adquisición de conocimiento afecta positivamente en los niveles de innovación presente en las empresas, así como que existe un enlace positivo entre el conocimiento de mercado o el conocimiento adquirido por los empleados con la innovación, por lo que se considera que invertir en investigación y desarrollo para generar conocimiento da lugar a la innovación dentro de las empresas (Darroch, 2005; Feldman, 1988; Tidd, 2001).

\section{Innovación}

La innovación, ha sido descrita como un ingrediente esencial para que las empresas puedan mantenerse competitivas y conseguir ventajas de largo plazo, siendo también un elemento crítico reconocido por otras ciencias para medir el creciemiento (Caravaca, González, \& Silva, 2005; 
Darroch \& McNaughton, 2002; Méndez, 2002). Se definen distintos tipos de innovación, dónde se destacan (a) la innovación radical que implica grandes cambios en la forma de hacer las cosas generalmente en un periodo corto y (b) la innovación incremental que genera cambios graduales en la forma de hacer las cosas; no existen muchos estudios que destaquen las diferencias entre estos tipos de innovación (Darroch \& McNaughton, 2002), es importan identificar los tipos de innovación debido a que cada tipo probablemente requiera distintos recursos y competencias esenciales (Rodríguez \& Vargas, 2004).

La innovación incremental, se representa como una extensión o modificación de productos existentes, la empresa mejora sus competencias internas existentes para construir conocimiento sobre lo ya conociendo, las ideas provienen del mercado, por lo que categorizan a la empresa como orientada hacia el mercado; están mayormente alineadas a las necesidades expresadas por los consumidores, este tipo de innovación es aplicada generalmente por las empresas que se definen como orientadas al mercado (Darroch \& McNaughton, 2002).

La innovación radical, destruye a la competencia, generalmente se requiere una distinta práctica gerencial, este tipo de innovación es originado por científicos, generalmente ponen a las empresas en riesgo, puesto que se originan productos difíciles de comercializar; es importante para el éxito a largo plazo debido a que envuelve el desarrollo y la aplicación de nueva tecnología las cuales podrían cambiar las estructuras existentes de mercado, abre nuevas oportunidades de innovación incremental, generalmente ignora las necesidades expresadas por los consumidores; este tipo de innovación es aplicada generalmente por las empresas que se definen como orientadas a la tecnología (Darroch \& McNaughton, 2002).

\section{Conclusiones}

La diseminación y la capacidad de actuar frente al conocimiento, han sido debatidas como los principales componentes que impactan la creación de una ventaja competitiva sostenible, como la innovación (Darroch \& McNaughton, 2002). Para ser innovador es necesario tener una orientación de mercado, así como estar en pro de la tecnología, tener procesos que permitan obtener información de los clientes de forma permanente; esto implica una serie de esfuerzos para combinar la tecnología y los procesos de tal forma que representen ventaja competitiva (Du Plessis, 2007). Factores que podrían servir como predictores para el incremento de innovación dentro de las empresas: (a) ser flexible y oportunista, (b) tener sensibilidad a la información sobre cambios en el mercado, (c) acciones que se toman frente al conocimiento sobre tecnología, (d) usar tecnología para difundir el conocimiento, (e) tener capital humano con perfil en ciencia y tecnología, (f) estar enfocado al mercado para adquirir conocimiento (Anantatmula, 2008; Darroch \& McNaughton, 2003; Kuo et al., 2011).

Es importante recalcar que la gestión del conocimiento que afecta el desarrollo de la innovación dentro de las empresas, estaría relacionada de forma positiva con el estilo de liderazgo que ejercen los gerentes en las organizaciones (Adams et al., 2006; Bassett-Jones, 2005; Tidd, 2001).

\section{Bibliografía}

Adams, R., Bessant, J., \& Phelps, R. (2006). Innovation management measurement: A review. International Journal of Management Reviews, 8(1), 21-47.

Anantatmula, V. S. (2008). Leadership role in making effective use of km. VINE, 38(4), 445-460. 
Bassett-Jones, N. (2005). The paradox of diversity management, creativity and innovation. Creativity and innovation management, 14(2), 169-175.

Blázquez Manzano, A. (2013). Un modelo de pensamiento estratégico para favorecer la gestión del cambio en las organizaciones. AD-minister (23), 9-24.

Caravaca, I., González, G., \& Silva, R. (2005). Innovación, redes, recursos patrimoniales y desarrollo territorial. EURE (Santiago), 31(94), 5-24.

Darroch, J. (2005). Knowledge management, innovation and firm performance. Journal of knowledge management, 9(3), 101-115.

Darroch, J., \& McNaughton, R. (2002). Examining the link between knowledge management practices and types of innovation. Journal of intellectual capital, 3(3), 210-222.

Darroch, J., \& McNaughton, R. (2003). Beyond market orientation: Knowledge management and the innovativeness of New Zealand firms. European journal of Marketing, 37(3/4), 572-593.

Du Plessis, M. (2007). The role of knowledge management in innovation. Journal of knowledge management, 11(4), 20-29.

Feldman, S. P. (1988). How organizational culture can affect innovation. Organizational dynamics, 17(1), 57-68.

Gloet, M., \& Terziovski, M. (2004). Exploring the relationship between knowledge management practices and innovation performance. Journal of Manufacturing Technology Management, 15(5), 402-409.

Jantunen, A. (2005). Knowledge-processing capabilities and innovative performance: An empirical study. European Journal of Innovation Management, 8(3), 336-349.

Jover, J. N., Montalvo, L. F., \& Ones, I. P. (2006). La gestión del conocimiento, la ciencia, la tecnología y la innovación en la nueva universidad: Una aproximación conceptual. Pedagogía Universitaria, 11(2).

Kuo, R.-Z., Lai, M.-F., \& Lee, G.-G. (2011). The impact of empowering leadership for kms adoption. Management Decisión, 49(7), 1120-1140.

Méndez, R. (2002). Innovación y desarrollo territorial: Algunos debates teóricos recientes. EURE (Santiago), 28(84), 63-83.

Rodríguez, F. M., \& Vargas, M. (2004). Hacia una caracterización de los procesos de innovación en la industria colombiana. Los resultados de un estudio de casos. Academia. Revista Latinoamericana de Administración (33), 5-33.

Tidd, J. (2001). Innovation management in context: Environment, organization and performance. International Journal of Management Reviews, 3(3), 169-183. 